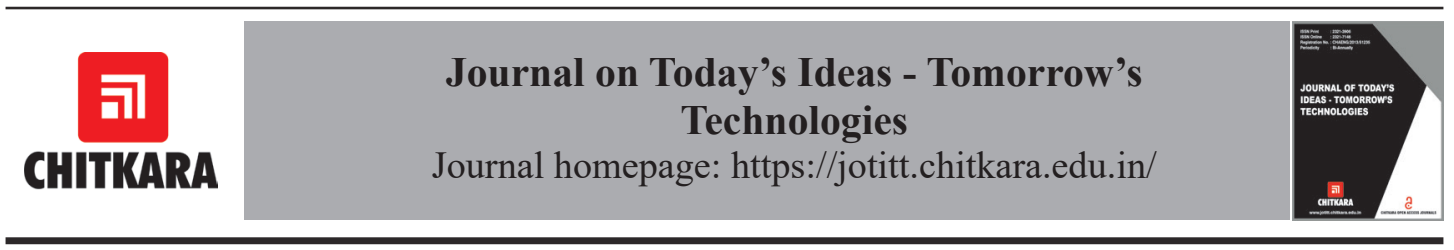

\title{
Economic and Easy Do It Yourself (DIY) Safety Kit
}

\author{
Yukta Chauhan \\ Chitkara University Institute of Engineering \& Technology, Chitkara University, Punjab-140401, India \\ Email: yukta1582.cse19@chitkara.edu.in
}

\section{ARTICLE INFORMATION}

Received: June 10, 2020

Revised: June 30, 2020

Accepted: July 13, 2020

Published online: December 03, 2020

Keywords:

DIY safety kit, Making

COVID-19 safety kit at home,

Biodegradable bag for making

safety kit

\begin{abstract}
The main objective of this paper is to present a cost effective and efficient safety kit in order to help fulfill the scarcity of PPE kits during COVID-19 pandemic. These days it is quite essential to wear safety gears like masks, gloves, face shields etc. resulting in shortage of these items at some places and the second problem is that certain sections of people cannot afford to purchase these items. This paper presents methods to create cheap yet effective safety kits at home. The kit will require easily available material like cloth bag, biodegradable trash bag, polythene bag etc. and items like gown, cap, masks, and gloves can be made using these materials.
\end{abstract}

DOI: $10.15415 /$ jotitt.2020.81002

\section{Introduction}

The project idea has been evolved from the urgent need of safety equipments for common people and the scarcity of PPE kits in India during this pandemic of COVID-19 [1]. In addition, some of the safety kits that are available in the market have negative effects like they are expensive and cost about INR 200 or more [2], they cause skin problems [3] and they are not made from biodegradable material and therefore are harmful for environment [4]. This paper has attempted to address some of these abovementioned issues. Safety kit proposed in this work is going to be completely biodegradable, reusable, cheap, skin friendly and breathable and it will contain an overall gown, a mask, a cap, gloves, organic non-alcoholic sanitizer and paper soap.

\section{Methodology}

In this section, the methods for making each of the aforementioned items of the safety kit have been discussed.

\subsection{Material and Methods for Making Gown, Mask, Cap and Gloves}

Cloth based shopping bag, biodegradable trash bags, polythene bags, polythene sheets or any such material (as in Fig. 1) are being proposed for making gowns, masks, caps and gloves. Nowadays cloth-based shopping bags are readily available and we recommend that cloth based 

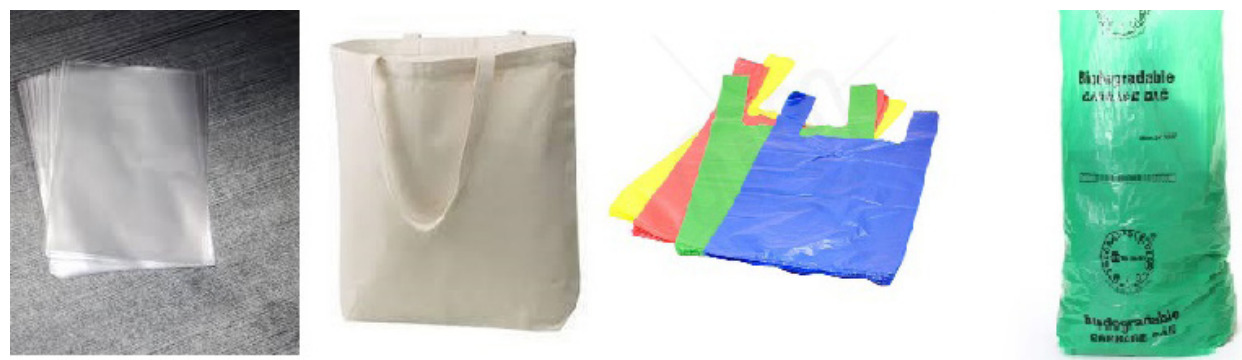

Figure 1: Cloth based shopping bag/ biodegradable trash bags/ polythene bags/ polythene sheets or any such material

shopping bags should be used for making these safety items. In order to make an overall disposable gown, the recommended biodegradable sheet needs to be cut into different parts and then sewed together. For making a head cap, biodegradable sheet is cut into circular shape as per the size of the head and a thread is attached along the circumference of the circular sheet. The ends of the thread are left open and pulling them will tighten the cap, in addition an elastic band can also be used to make a bouffant cap.

Gloves are made by cutting a sheet in a shape of hands, and in a slightly bigger size in comparison to the intended hands. For each hand two sheets need to be cut in exactly similar sizes and shapes and both the sheets are then glued to make a glove. In order to make a face mask, biodegradable sheet needs to be cut into rectangular shape of appropriate size in order to cover mouth and face properly. This is followed by making two to three pleats and gluing the ends of the pleats. Finally, a string or a thread is glued to the pleated mask.

\subsection{Material and Method for Making Paper Soap}

In order to make paper soap any type of paper be it a normal unruled paper, butter paper or wax paper may be used. The intended paper needs to be placed in a tray and liquid soap is poured into it. Soap should be evenly spread all over the paper and paper is then dried in sun. The sheet of paper soap is then cut into appropriate sizes for storing and for use.

\subsection{Material and Method for Making Non-alcoholic Sanitizer}

The proposed non-alcoholic sanitizer is a good alternative to alcoholic sanitizer, and it has good antibacterial properties. Proposed sanitizer is made using vinegar(acetic acid) which has good antibacterial properties [5]. In order to make sanitizer using vinegar, $30 \mathrm{ml}$ of white vinegar is mixed with 1 tablespoon of baking soda which is a good disinfectant [6]. A teaspoon of turmeric and lemon that respectively have good antiseptic and antibacterial property is added to the mixture $[7,8]$. Half tablespoon each of glycerine and coconut oil is added which will help in preventing skin damage. The mixture is then poured into a bottle and can be used as hand sanitizer.

\section{Results and Discussion}

The proposed safety kit is going to benefit both the consumers as well as manufacturers. Since it is very simple to make kit, marginal and lowincome group people looking for employment in these difficult times of COVID-19 can make them and sell them. NGOs can aid them in selling these kits through local chemists, retailers as well as through e-commerce platforms. Consumers will be benefitted as this is going to be a lowcost product, with the cost of complete kit is no more than INR 40.

\section{Conclusion}

Safety kit discussed in this paper is going to be extremely useful during the COVID-19 pandemic. 
It is safe to use, safe to dispose, reusable and cost-effective kit that can be made in no time. It could prove to be a source of employment for low- and marginal-income group people and since it is low cost it will be within the reach of every consumer. A kit like this can help people stay safe during this COVID-19 pandemic.

\section{References}

[1] P. Sarda. India's PPE crisis puts workers in the line of fire. Forbes India, April 2020. [Online] Available: https://www. forbesindia.com/article/coronavirus / indias-ppe-crisis-puts-workers-in-the-lineof-fire/59073/1 [Accessed: May, 2020].

[2] T. Dhaliwal and Y. Kaushal. Patients coughing up Rs. 1,800-5,00O for PPE kits of health workers in Chandigarh tricity's private hospitals Overpriced PPE kits. Hindustan Times, May, 2020. [Online] Available: https://www.hindustantimes. com/chandigarh/patients-coughing-up-1800-5-000-for-ppe-kits-of-health-workersin- chandigarh-tricity-s-private-hospitals/ story-S9cHQd2TuqbwMiV7GLaaoK.html [Accessed: May 2020].

[3] S. Chadha. Rash, sweat, bruises: Wearing PPE for hours causing skin infection. May 2020. [Online] Available: https://fit.thequint.com/ coronavirus/ppe-causes-rashes-sweat-anddiscomfort-doctors-share-experiences-duringcoronavirus-care [Accessed: May 2020].
[4] L. Tenenbaum. The amount of plastic waste is surging because of the coronavirus pandemic April 2020. [Online] Available: https://www.forbes.com/sites/ lauratenenbaum/2020/04/25/plastic-wasteduring-the-time-of-covid-19/?sh=4da47bo47e48 [Accessed: May 2020].

[5] D. Drake. Antibacterial activity of baking soda. Compend Contin Educat Dent Supply, vol. 18, no. 21, pp. 17-21,1997.

[6] P. Gul and J. Bakht. Antimicrobial activity of turmeric extract and its potential use in food industry. Journal of Food Sciene and Technology, vol. 52, no. 4, pp. 2272-2279, 2015. https://doi.org/10.1007/s13197-013-1195-4

[7] S. Bakir, D. Devecioglu, S. Kayacan. Investigating the antioxidant and antimicrobial activities of different vinegars. European Food Research and Technology, vol. 243, pp. 2083-2094, 2017. https://doi.org/10.1007/s00217-017-2908-0

[8] M. Viuda-Martos Y. Ruiz-Navajas J. Fernández-López J. Perez-Álvarez. Antibacterial Activity of Lemon (Citrus Lemon L.), Mandarin (Citrus Reticulata L.), Grapefruit (Citrus Paradisi L.) And Orange (Citrus Sinensis L.) Essential Oils. October 2008. https://doi.org/10.1111/j.1745-4565.2008.00131.x

This article has been published as a part of Special regular issue of Journal on Today's Ideas Tomorrow's Technologies. Most of the articles in this issue are the papers for which the original ideas were presented at NOVATE 2020 (https://www.chitkara.edu.in/novate), a competition organized by Chitkara University and supported by Ministry of Electronics and Information Technology, Govt. Of India, Institution's Innovation Council, NSTEDB, Govt. Of India and FICCI. Student participants have written most of these articles. Some basic editing work has been done at the editorial level, but same have not been corrected very rigorously so as to preserve basic touch of student's writing. 


\section{旬 \\ ChItKARA \\ Journal on Today's Ideas - Tomorrow's Technologies \\ Chitkara University, Saraswati Kendra, SCO 160-161, Sector 9-C, Chandigarh, 160009, India}

Volume -8, Issue-1

June 2020

ISSN 2321-3906

Copyright: [C 2020 Yukta Chauhan] This is an Open Access article published in Journal on Today's Ideas - Tomorrow's Technologies by Chitkara University Publications. It is published with a Creative Commons Attribution- CCBY 4.0 International License. This license permits unrestricted use, distribution, and reproduction in any medium, provided the original author and source are credited. 